\title{
Association of preadmission metformin use and mortality in patients with sepsis and diabetes mellitus: a systematic review and meta-analysis of cohort studies
}

\author{
Huoyan Liang ${ }^{1+}$, Xianfei Ding ${ }^{1+}$, Lifeng $\mathrm{Li}^{2}$, Tian Wang ${ }^{2}$, Quancheng Kan ${ }^{3}$, Lexin Wang ${ }^{4}$ and Tongwen Sun ${ }^{{ }^{*}}$ (D)
}

\begin{abstract}
Background: Recent studies have reported that preadmission metformin users had lower mortality than non-metformin users in patients with sepsis and diabetes mellitus; however, these results are still controversial. Therefore, we conducted a systematic review and meta-analysis of published observational cohort data to determine the association between preadmission metformin use and mortality in septic adult patients with diabetes mellitus.

Methods: The MEDLINE, EMBASE, and Cochrane CENTRAL databases were searched from their inception to September 30, 2018. Cohort studies that evaluated the use of metformin in septic adult patients with diabetes mellitus were included. The quality of outcomes was evaluated using the Newcastle-Ottawa Scale (NOS). The inverse variance method with random effects modelling was used to calculate the pooled odds ratios (ORs) and $95 \% \mathrm{Cls}$.

Results: Five observational cohort studies (1282 patients) that were all judged as having a low risk of bias were included. In this meta-analysis, metformin use was associated with a significantly lower mortality rate (OR, 0.59; 95\% Cl, 0.43-0.79, $P=0.001$ ).

Conclusions: This meta-analysis indicated an association between metformin use prior to admission and lower mortality in septic adult patients with diabetes mellitus. This finding suggested that the possible effect of metformin should be evaluated in future clinical trials.
\end{abstract}

Keywords: Metformin, Mortality, Sepsis, Diabetes mellitus, Meta-analysis, Systematic review

\section{Introduction}

Sepsis is a life-threatening organ dysfunction caused by dysregulated host responses to infection [1]. Because the high mortality due to sepsis remains a major medical problem $[2,3]$, exploring the mechanism of its development is particularly important. To date, the exact mechanism remains unclear, but it is widely postulated that the release of inflammatory factors by innate immune cells plays an important role in sepsis disease pathogenesis $[4,5]$. Interestingly, recent studies indicated that the

\footnotetext{
* Correspondence: suntongwen@163.com

†Huoyan Liang and Xianfei Ding contributed equally to this work.

${ }^{1}$ General ICU, The First Affiliated Hospital of Zhengzhou University, Henan

Key Laboratory of Critical Care Medicine, Zhengzhou 450052, China

Full list of author information is available at the end of the article
}

active molecular responses in inflammation require intensive metabolic support, and modulation of the metabolic pathways could become a novel strategy to restrict inflammatory diseases [6].

Metformin is a reagent with extensive and strong metabolic regulatory activities, and it is often used as a first-line antidiabetic drug for the treatment of type 2 diabetes $[7,8]$. In addition to its well-known hypoglycaemic activities, increasing evidence has suggested that metformin inhibits the expression of pro-inflammatory factors in vitro and ameliorates inflammatory injuries in vivo [9-14]. The mechanisms underlying the pharmacological effects of metformin remain unknown. It has been suggested that metformin inhibits the activity of the mitochondrial respiratory complex, which decreases the generation of ATP and activates 
adenosine 5'-monophosphate (AMP)-activated protein kinase (AMPK) [15]. Furthermore, several studies have suggested that AMPK activation by drugs or small molecular compounds protects against experimental sepsis in animals [16-18], demonstrating that AMPK plays an irreplaceable role in the pathogenesis of sepsis. Moreover, AMPK has been regarded as a major target mediating the effects of metformin.

Given the evidence of AMPK activation by metformin, metformin is a potential treatment for sepsis. In recent years, some studies $[19,20]$ have suggested that in patients with sepsis and diabetes mellitus, the preadmission metformin users had higher lactate levels but lower mortality than the non-metformin users. Geen et al. [21] reported similar lactate levels between preadmission metformin users and non-metformin users, but metformin users still had significantly lower mortality than nonusers. However, some studies $[22,23]$ showed that preadmission metformin use had no significant effect on mortality compared with non-metformin use in septic patients with diabetes mellitus. Therefore, it is necessary to consolidate the available information to assess whether metformin is beneficial for improving the outcomes of sepsis in patients with diabetes mellitus.

\section{Methods}

\section{Search strategy}

The methods complied with the meta-analysis of Observational Studies in Epidemiology guidelines [24], and the study profile is presented in Fig. 1 . We searched the MEDLINE, EMBASE, and Cochrane CENTRAL databases for English language articles published from the inception of the database to September 30, 2018. A combination of $\mathrm{MeSH} / \mathrm{Em}$ tree and title/abstract keywords was used. The search terms were "metformin", "sepsis," "severe sepsis", "septic shock", "mortality", and "death".

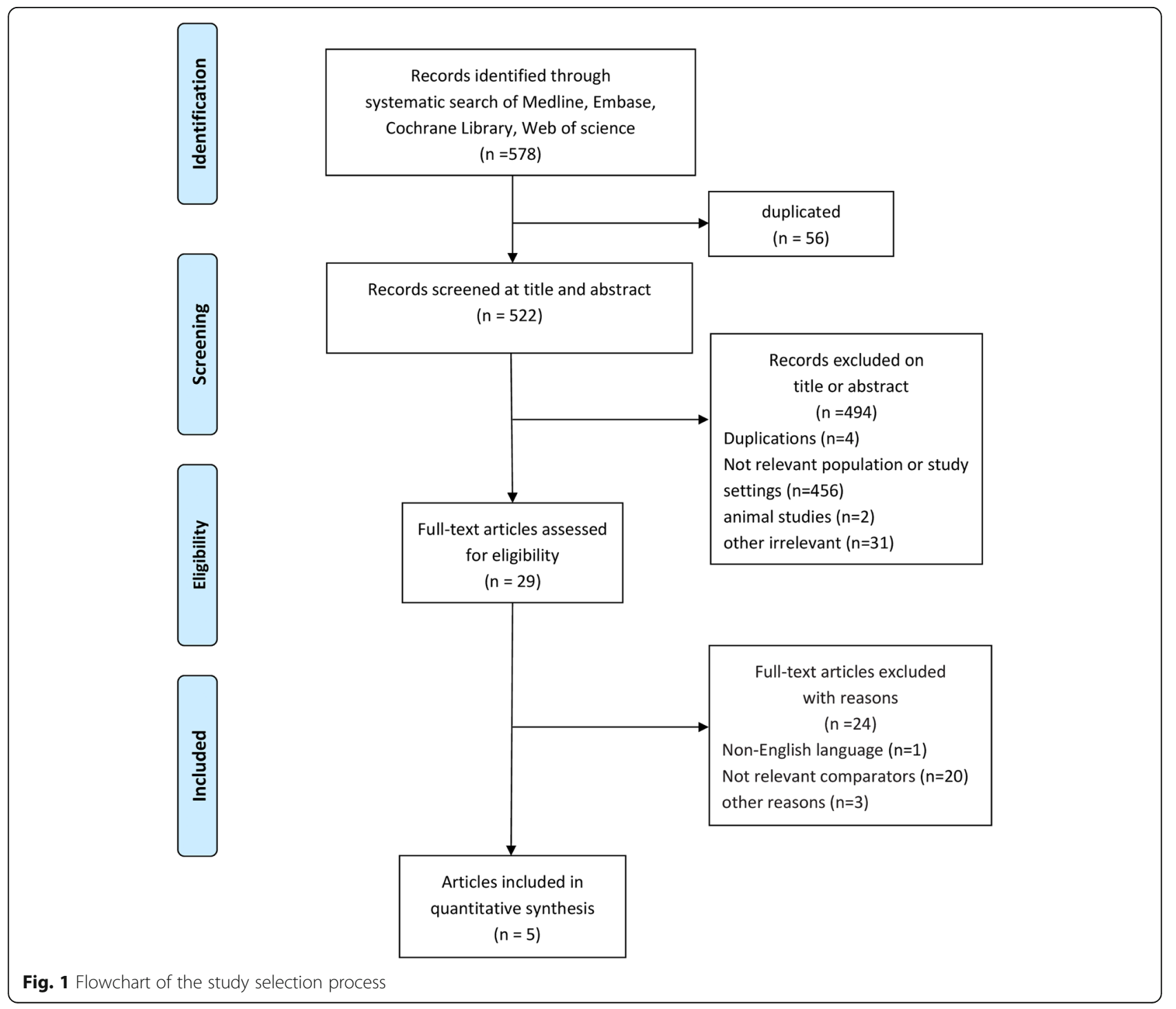




\section{Eligibility criteria}

Studies were considered suitable for inclusion in this meta-analysis if (1) they enrolled septic patients with diabetes mellitus who used metformin, (2) the comparative arms were septic patients with or without diabetes mellitus, (3) they measured the mortality of metformin users and non-metformin users, (4) all the patients were adults, (5) they were observational studies from all settings (emergency department, hospital ward, or ICU), and (6) they were written in English. If the studies lacked outcome data or provided only the mortality of patients with sepsis complicated with other illnesses, they were excluded. If the full text could not be retrieved or if the article was a commentary or a review, it was excluded.

\section{Selection of studies and data extraction}

All the available data were extracted from each study by two investigators independently according to the aforementioned inclusion criteria, and any differences were resolved by discussion with a third investigator. The following data were collected from each study: the name of the first author, publication year, country where the research was performed, study design, number of patients, sex, mean age, primary outcome, follow-up, and unadjusted and adjusted odds ratios (ORs) and 95\% CIs of the primary outcome.

\section{Risk of bias assessment}

The risk of bias was assessed for each outcome in all included studies using the Newcastle-Ottawa Scale (NOS) for cohort studies [25]. A maximum of nine points were awarded based on the cohort selection (maximum four points), the comparability of the cohort design and analysis (maximum two points), and the adequacy of the outcome measures (maximum three points); seven to nine points was considered high quality [25].

\section{Statistical analysis}

The outcome of interest was the mortality of patients with sepsis with preadmission metformin use vs. non-metformin use. This meta-analysis used the pooled effect of each outcome. To investigate the heterogeneity between studies, we used a fixed effects model to calculate odds ratios (ORs) and $95 \%$ CIs for each outcome. Heterogeneity was assessed using $I^{2}$ and $P$ values, and the percentage of variability that was due to heterogeneity rather than sampling error (13, 14) was considered moderate when $I^{2}$ equalled $51-74 \%$ and high when $I^{2}$ was greater than or equal to $75 \%$. Begg's funnel plot [26] and Egger's linear regression [27] were used to assess potential publication bias. Funnel plots were visually assessed for asymmetry. For Egger's tests, $P<0.1$ indicated a significantly small study size. All statistical analyses were performed using STATA 14.0 (College Station, Texas 77845, USA, Serial number: 401406267051).

\section{Results}

\section{Study selection}

We initially identified 578 records, and 56 articles remained after duplicates were removed. After preliminary screening by title and abstract, 29 cohort studies were identified that appeared to address issues potentially related to the primary study question. However, only 5 articles [19-23] enrolling 1282 patients were ultimately included in this meta-analysis after the study selection process (Fig. 1).

\section{Study characteristics}

All included articles were observational cohort studies that reported patients with sepsis and diabetes mellitus who used metformin preadmission [19-23]. Three studies [19, 20, 23] reported in-hospital mortality as the primary outcome, and the remaining two studies $[21,22]$ reported 28-day mortality as the primary outcome. Then, we extracted the unadjusted and adjusted ORs and 95\% CIs of the primary outcome data. If missing, the data were calculated based on the raw data provided in the study. Baseline information about the analysed studies is presented in Table 1.

\section{Risk of bias assessment}

The five eligible studies were retrospective cohort studies, all of which had greater than or equal to seven points and showed a low risk of bias according to the NOS. Specific details of the risk of bias in the included studies are reported in Table 2.

\section{Effects of metformin on outcomes}

In the five included cohort studies (1282 patients), preadmission metformin users had significantly lower mortality than nonusers among patients with sepsis (OR, 0.59; 95\% CI, 0.43-0.79, $P=0.001$ ) (Fig. 2) [19-23]. Because few studies have investigated the association of metformin with the length of hospital stay and other outcomes, a meta-analysis of secondary outcomes was not conducted.

\section{Sensitivity analyses}

Because all included studies were observational cohort studies with a low risk of bias (Table 1), a sensitivity analysis based on the methodological criteria was not conducted. A sensitivity analysis was performed only to assess the influence of any one study on the pooled OR and $95 \%$ CI by omitting one individual study at a time. Our findings showed that the results were robust and reliable (Fig. 3). In addition, based on the analysis of metformin users stratified by age, the heterogeneity of the overall estimate in each subgroup was not significant.

\section{Evaluation of publication bias}

Funnel plots (Fig. 4) and Egger's regression asymmetry tests were performed to evaluate the publication bias in 


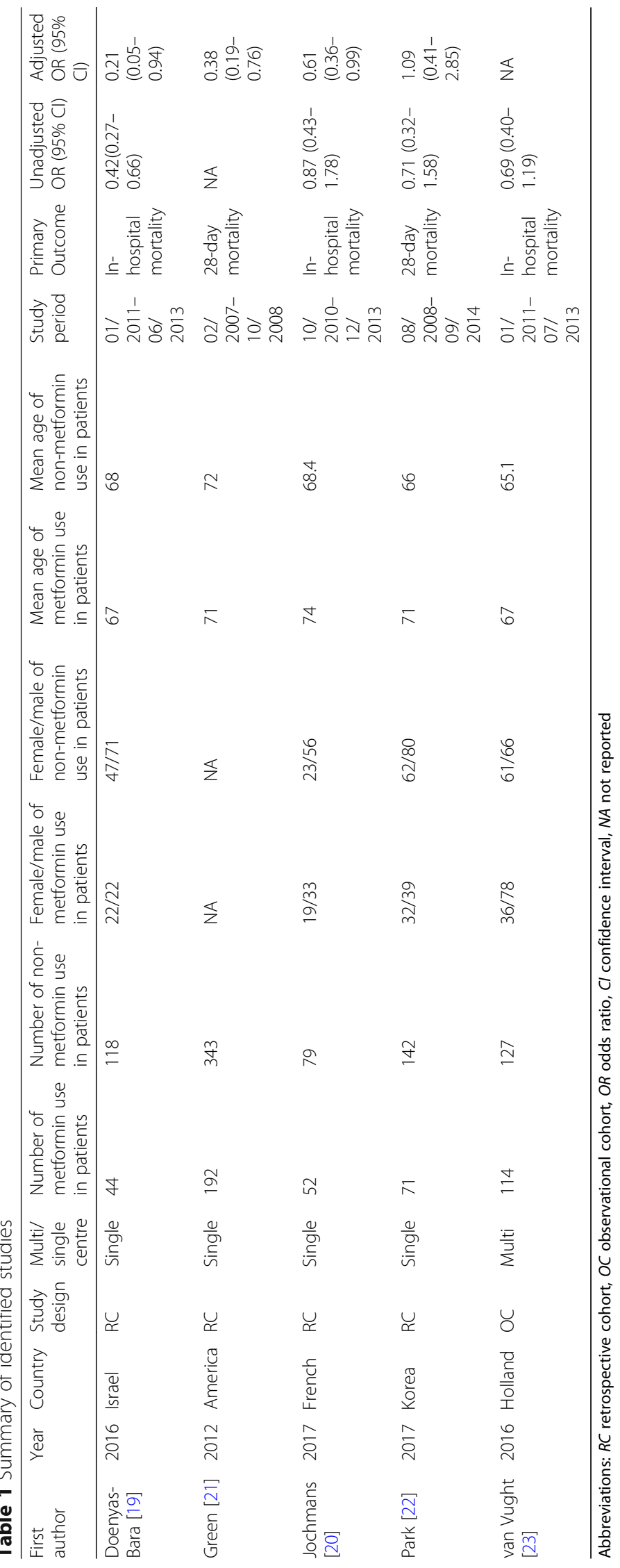




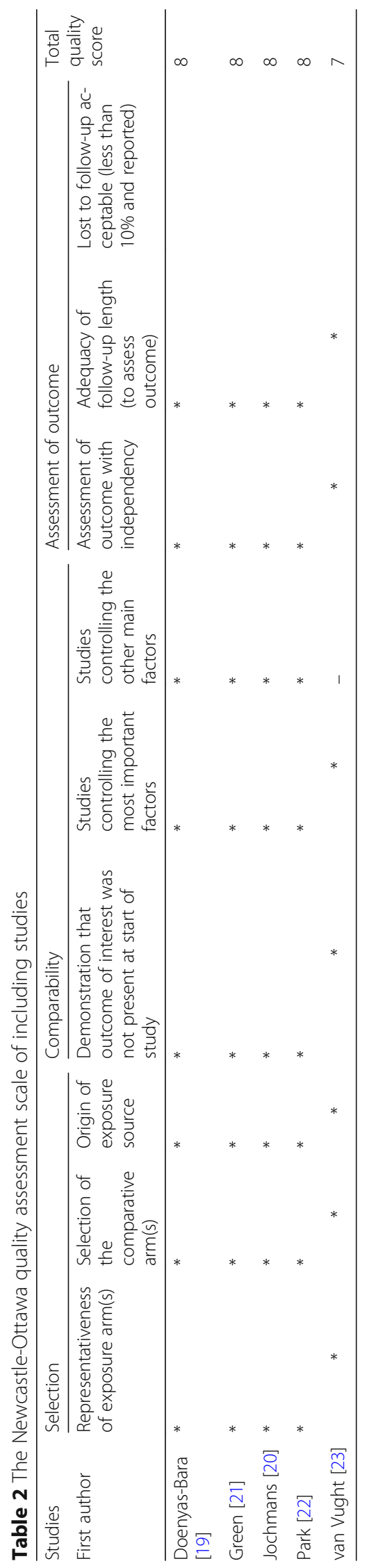




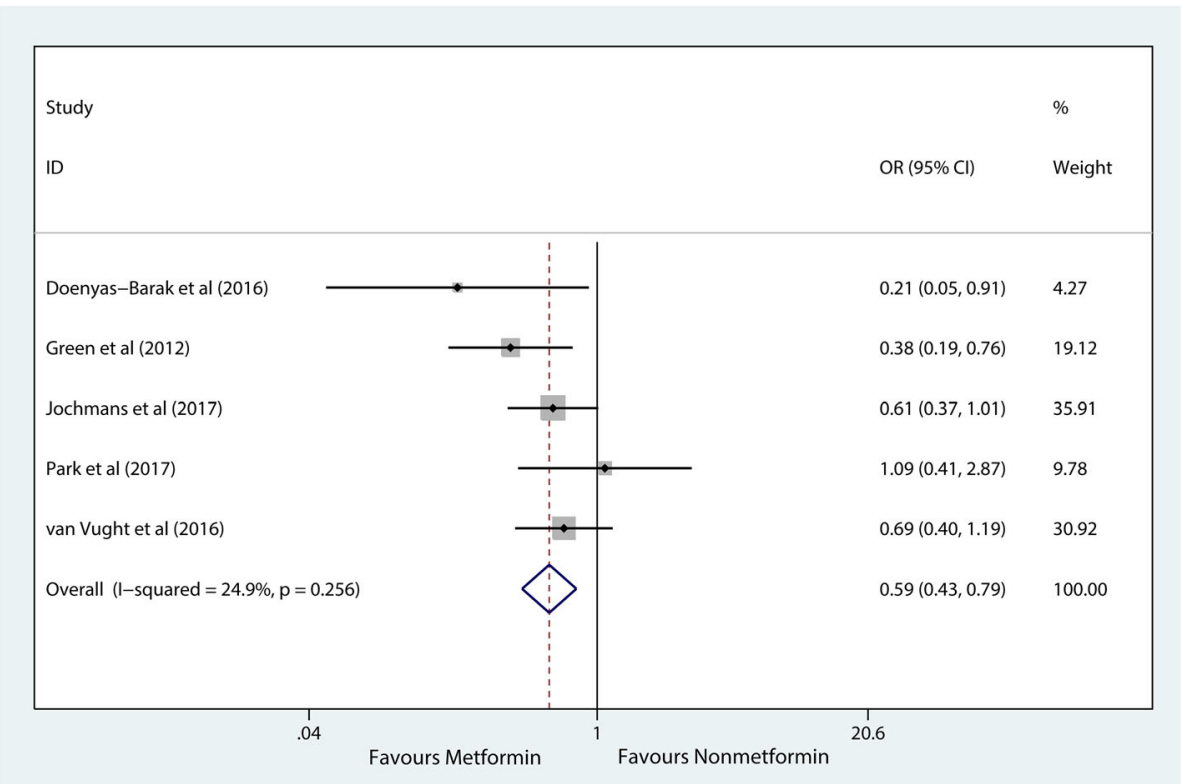

Fig. 2 Meta-analysis of the overall pooled odds ratios (ORs) of studies investigating the mortality outcomes of patients with sepsis with diabetes mellitus. Forest plot showing the significance of the association between metformin use and mortality in septic patients with diabetes according to the fixed effects model

these five studies evaluating mortality after metformin use in septic patients with diabetes mellitus. No significant publication bias was found $(P=0.570)$.

\section{Discussion}

This meta-analysis included 1282 patients and demonstrated that preadmission metformin users had lower mortality than nonusers in patients with sepsis. This is the first systematic review and meta-analysis to describe the association between preadmission metformin use and mortality in septic adult patients with diabetes mellitus. Our finding indicates that metformin may have therapeutic potential for patients with sepsis and diabetes mellitus.

A beneficial association between metformin and mortality has already been described in selected ICU patients with chronic heart failure and liver disease [28-31], and a retrospective study [31] of 17 Danish ICUs found that

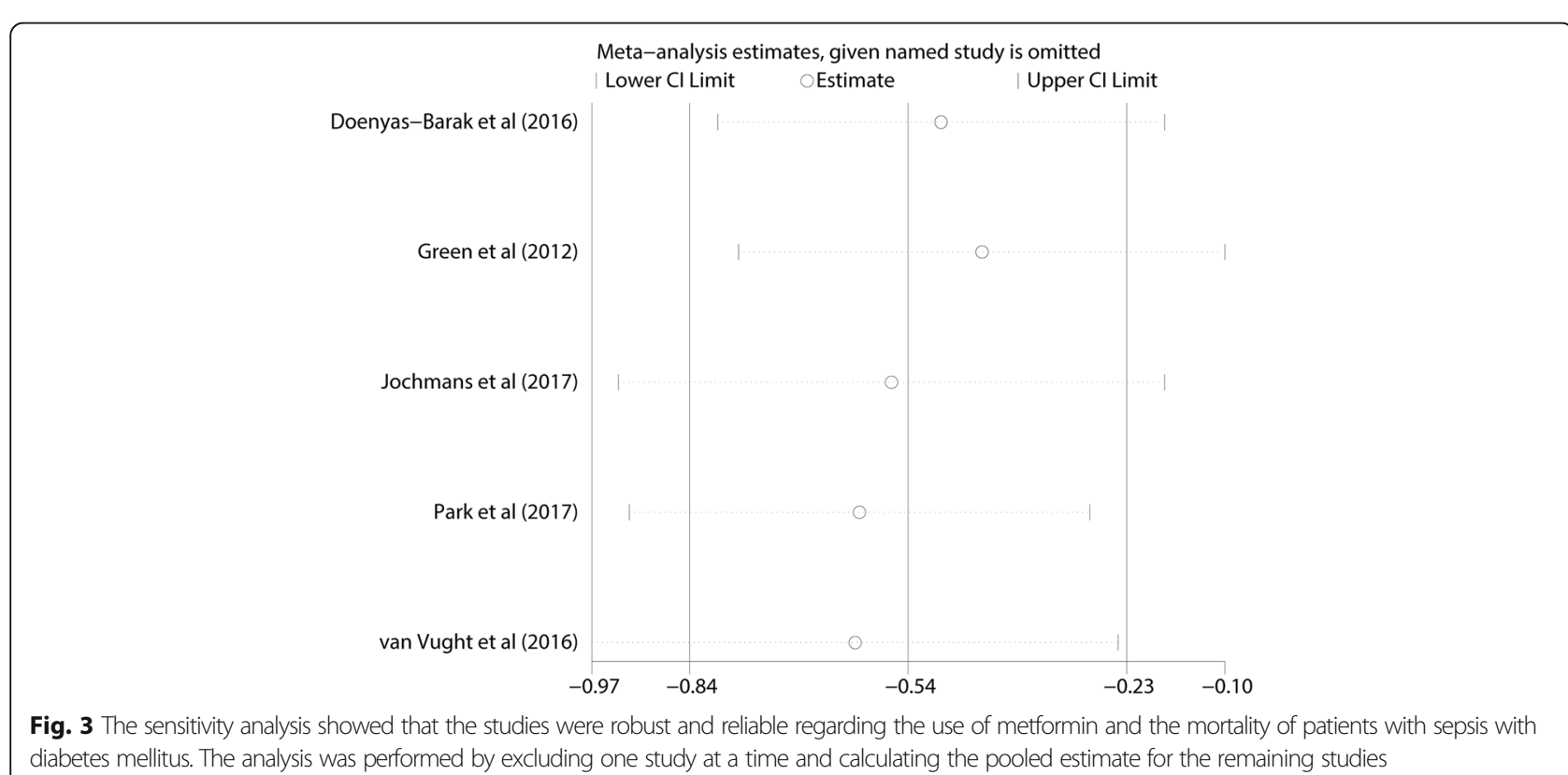




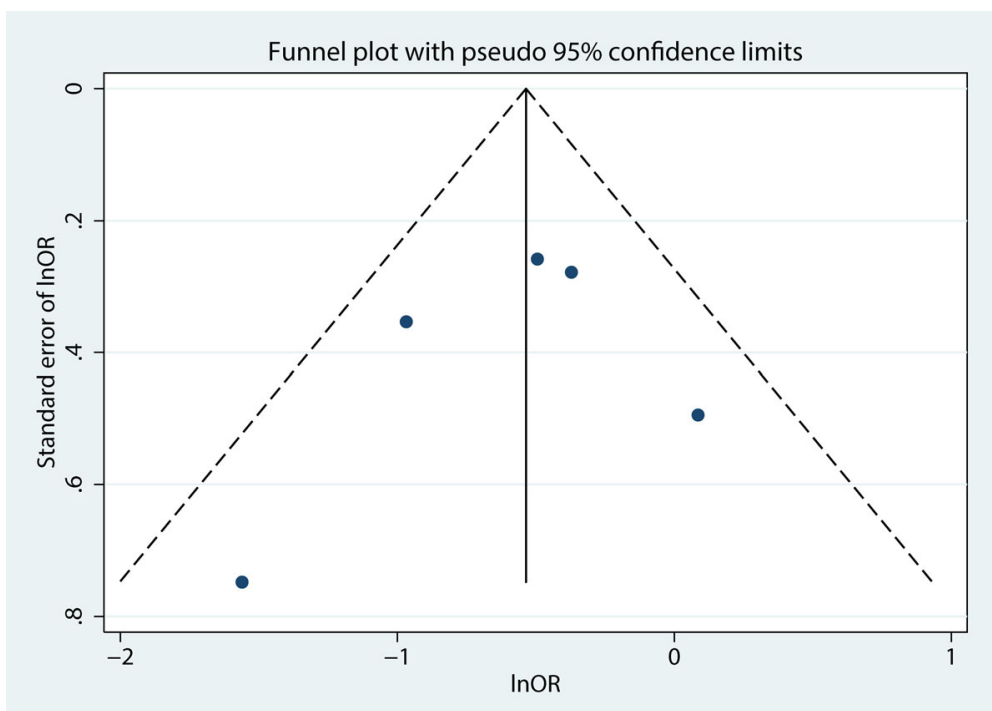

Fig. 4 Funnel plot evaluating mortality after the preadmission use of metformin in septic patients with diabetes mellitus

30-day mortality was lower in metformin users than in non-metformin users, which was the same result as that found in this meta-analysis. In addition, many experimental studies [32-37] have reported that metformin can ameliorate sepsis or endotoxaemia-induced injuries in various organs and many inflammatory diseases. For example, Wu et al. [38] reported that metformin can alleviate acute lung injury, and Liu et al. [13] showed that metformin can attenuate acute myocarditis. However, some studies $[39,40]$ have shown that metformin cannot decrease but instead increases the mortality rate of septic animals. And unfortunately, to date, the association between preadmission metformin use and mortality in septic patients with diabetes mellitus remains controversial. Some studies $[20,22,23]$ have shown that in patients with sepsis, preadmission metformin users were not significantly different from nonusers, whereas the studies by Green et al. [21] and Doenyas-Bara et al. [19] suggested that the mortality was significantly lower in preadmission metformin users than in nonusers among patients with sepsis. Therefore, this meta-analysis provides evidence to support that preadmission metformin use may decrease mortality in septic patients with diabetes mellitus.

The mechanism underlying the ability of metformin to decrease the mortality of septic patients remains unclear. Metformin may supply higher amounts of lactate, serving as an energetic carbon source, thus making energy available to ischaemic tissue; this hypothesis is consistent with the results of a study [41] that indicated that lactic acid is also a key energy source, like glucose, amino acids, and ketones. Furthermore, Friesecke et al. [42] and Protti et al. [43] suggested that lactic acidosis due to metformin use is associated with lower mortality compared to other forms of lactic acidosis, which may explain why preadmission metformin users had lower mortality than nonusers among patients with sepsis and diabetes mellitus. Moreover, the mechanism may also be related to the potential protective effects of metformin, including its anti-endotoxaemic, anti-inflammatory, and vasoactive properties [44-46]. Additionally, metformin has been shown to decrease the expression of nitric oxide synthase in animal models, which could ameliorate vasodilatation [47]. Most importantly, metformin induces the activation of AMPK, an enzyme that is a key cellular energy sensor that maintains energy homeostasis at the cellular and organismal levels [48]. Once activated, AMPK regulates cellular energy status, switching on the ATP-generating pathways and switching off the ATP-consuming pathways [49]. Furthermore, some studies $[50,51]$ have shown that metformin may possess antimicrobial effects, which may be the primary mechanism involved in the beneficial effects on sepsis. As a result, cellular function is improved under stressful conditions, leading to improved cardiovascular function and hypoxia. These potential protective effects of metformin use may significantly ameliorate the prognosis of septic patients, but this association requires further study.

Meta-analysis is a method used for the comprehensive statistical analysis of multiple studies on a topic. When the difference between the outcomes of each study is greater than expected, statistical heterogeneity is present in the pooled results of the meta-analysis. The four included cohort studies had a high degree of heterogeneity, reflecting differences in these studies. In this meta-analysis, the risk of bias assessment of the included 
studies showed a low risk bias. Therefore, the statistical heterogeneity was not considered methodological heterogeneity. The heterogeneity may be derived from many causes, such as the small sample sizes, the different initial lactate levels and the use of other antidiabetic medications.

This meta-analysis has several strengths. In the study by Doenyas-Bara et al. [19], the metformin users had more severe diabetes mellitus at admission than the non-metformin users, thus providing additional strong evidence to support the outcome that metformin users may have lower mortality among patients with sepsis and diabetes mellitus. Second, the risk of bias assessment used the NOS, which showed a low risk of bias among the included studies. Third, we used the random effects model with generic inverse variance, and we extracted the adjusted ORs and 95\% CIs so that the pooled estimate of the OR for the effect of metformin on mortality could be computed. Finally, the outcome of the sensitivity analysis showed that this result was robust and reliable.

However, this study has important limitations. Although we did our best to conduct a comprehensive search of the relevant literature, only four studies met our inclusion criteria, so the conclusion may need more original studies for confirmation. Second, the sample size of the septic patients with diabetes mellitus was small, suggesting that the result may not be reliable. Nevertheless, the robustness of our conclusions was supported by the result of the risk of bias assessment. Third, the outcome of this meta-analysis may be confounded by the fact that the inclusion criteria of the four studies varied considerably. Fourth, we studied the relationship between metformin use prior to admission and mortality in septic patients with diabetes mellitus, so it is not clear whether metformin is beneficial during the hospital stay for septic patients with diabetes mellitus previously untreated with metformin; therefore, further studies are needed. Fifth, although septic patients with diabetes may benefit from preadmission metformin use, the effects and safety of metformin treatment initiation and continuation in patients who are critically ill remain to be further clarified. Finally, there were no randomised controlled trials included in this meta-analysis, which is a limitation even though all the included retrospective cohort studies had a low risk of bias.

\section{Conclusions}

This is the first systematic review and meta-analysis to describe the association of preadmission metformin use and mortality in septic adult patients with diabetes mellitus. The results suggest that in septic adult patients with diabetes mellitus, preadmission metformin use is associated with lower mortality. However, the current conclusions need to be further supported by more high-quality original studies.

\section{Abbreviations}

AMPK: Adenosine 5'-monophosphate (AMP)-activated protein kinase; Cl: Confidence interval; NOS: Newcastle-Ottawa Scale; OR: Odds ratio; $\mathrm{RC}$ : Retrospective cohort

\section{Acknowledgements \\ We would like to thank CYW and HYL for their help with this study and the Chinese Evidence Based Medicine Center, West China Hospital, Sichuan University, for providing the Stata 14.0 statistical software.}

\section{Funding}

This study was supported by the Natural Science Foundation of Henan Province (Grant No. 182300410369) and Science and Technology Innovation Talents in Universities of Henan Province (Grant No. 16IRTSTHN021), Scientific and Technological Innovation leaders in Central Plains (Grant No.

194200510017), Provincial Ministry Co-construction Project from Medical Scientific and Technological Research Program of Henan Province (Grant No. SBGJ2018020), the "51282" Project Leaders of Scientific and Technological Innovative Talents from Health and Family Planning Commission in Henan Province (2016-32).

\section{Availability of data and materials}

The datasets used and/or analysed in the present study are available from the corresponding author on reasonable request.

\section{Authors' contributions}

All the authors contributed substantially to the work presented in this article. TWS, HYL, and XFD conceived the study. LFL and TW contributed to the data interpretation. HYL and XFD contributed to the study protocol and wrote the article. QCK, LXW, and TWS revised the article. The corresponding author had full access to all of the data and the final responsibility for the decision to submit this article for publication. All authors read and approved the final manuscript.

\section{Ethics approval and consent to participate \\ Not applicable.}

\section{Consent for publication}

Not applicable.

\section{Competing interests}

The authors declare that they have no competing interests.

\section{Publisher's Note}

Springer Nature remains neutral with regard to jurisdictional claims in published maps and institutional affiliations.

\section{Author details \\ ${ }^{1}$ General ICU, The First Affiliated Hospital of Zhengzhou University, Henan Key Laboratory of Critical Care Medicine, Zhengzhou 450052, China. ${ }^{2}$ Biotherapy Center, The First Affiliated Hospital of Zhengzhou University, Zhengzhou 450052, China. ${ }^{3}$ Department of Pharmacy, The First Affiliated Hospital of Zhengzhou University, Zhengzhou 450052, China. ${ }^{4}$ School of Biomedical Sciences, Charles Sturt University, Wagga Wagga, NSW 2650, Australia.}

Received: 22 November 2018 Accepted: 6 February 2019 Published online: 18 February 2019

References

1. Shankar-Hari M, Phillips GS, Levy ML, Seymour CW, Liu VX, Deutschman CS, Angus DC, Rubenfeld GD, Singer M. Developing a new definition and assessing new clinical criteria for septic shock: for the Third International Consensus Definitions for Sepsis and Septic Shock (Sepsis-3). Jama. 2016; 315(8):775-87.

2. Czupryna P, Garkowski A, Moniuszko A, Pancewicz S, Ciemerych A, Zajkowska J. Patients with sepsis in infectious diseases department in years 1997-2010 - epidemiology and clinical features. Przegl Epidemiol. 2013;67(3): 429-34 535-428.

3. Vincent JL, Pereira AJ, Gleeson J, Backer D. Early management of sepsis. Clin Exp Emerg Med. 2014;1(1):3-7. 
4. Chousterman BG, Swirski FK, Weber GF. Cytokine storm and sepsis disease pathogenesis. Semin Immunopathol. 2017;39(5):517-28.

5. Bryant RV, Brain O, Travis SP. Conventional drug therapy for inflammatory bowel disease. Scand J Gastroenterol. 2015;50(1):90-112.

6. Prochnicki T, Latz E. Inflammasomes on the crossroads of innate immune recognition and metabolic control. Cell Metab. 2017;26(1):71-93.

7. Thomas I, Gregg B. Metformin; a review of its history and future: from lilac to longevity. Pediatr Diabetes. 2017;18(1):10-6.

8. Gray SG, McGuire TM, Cohen N, Little PJ. The emerging role of metformin in gestational diabetes mellitus. Diabetes Obes Metab. 2017;19(6):765-72.

9. Kuo CL, Ho FM, Chang MY, Prakash E, Lin WW. Inhibition of lipopolysaccharideinduced inducible nitric oxide synthase and cyclooxygenase-2 gene expression by 5-aminoimidazole-4-carboxamide riboside is independent of AMP-activated protein kinase. J Cell Biochem. 2008;103(3):931-40.

10. Zmijewski JW, Lorne E, Zhao X, Tsuruta Y, Sha Y, Liu G, Siegal GP, Abraham E. Mitochondrial respiratory complex I regulates neutrophil activation and severity of lung injury. Am J Respir Crit Care Med. 2008;178(2):168-79.

11. Kim SA, Choi HC. Metformin inhibits inflammatory response via AMPK-PTEN pathway in vascular smooth muscle cells. Biochem Biophys Res Commun. 2012;425(4):866-72.

12. Yuan H, Li L, Zheng W, Wan J, Ge P, Li H, Zhang L. Antidiabetic drug metformin alleviates endotoxin-induced fulminant liver injury in mice. Int Immunopharmacol. 2012;12(4):682-8.

13. Liu G, Wu K, Zhang L, Dai J, Huang W, Lin L, Ge P, Luo F, Lei H. Metformin attenuated endotoxin-induced acute myocarditis via activating AMPK. Int Immunopharmacol. 2017;47:166-72.

14. Koh SJ, Kim JM, Kim IK, Ko SH, Kim JS. Anti-inflammatory mechanism of metformin and its effects in intestinal inflammation and colitis-associated colon cancer. J Gastroenterol Hepatol. 2014;29(3):502-10.

15. An H, He L. Current understanding of metformin effect on the control of hyperglycemia in diabetes. J Endocrinol. 2016;228(3):R97-106.

16. Liu Z, Bone N, Jiang S, Park DW, Tadie JM, Deshane J, Rodriguez CA, Pittet JF, Abraham E, Zmijewski JW. AMP-activated protein kinase and glycogen synthase kinase 3 beta modulate the severity of Sepsis-induced lung injury Mol Med. 2016;21(1):937-50.

17. Mulchandani N, Yang WL, Khan MM, Zhang F, Marambaud P, Nicastro J, Coppa GF, Wang P. Stimulation of brain AMP-activated protein kinase attenuates inflammation and acute lung injury in Sepsis. Mol Med. 2015;21:637-44.

18. Escobar DA, Botero-Quintero AM, Kautza BC, Luciano J, Loughran P, Darwiche S, Rosengart MR, Zuckerbraun BS, Gomez H. Adenosine monophosphate-activated protein kinase activation protects against sepsisinduced organ injury and inflammation. J Surg Res. 2015;194(1):262-72.

19. Doenyas-Barak K, Beberashvili I, Marcus R, Efrati S. Lactic acidosis and severe septic shock in metformin users: a cohort study. Crit Care. 2016;20:10.

20. Jochmans S, Alphonsine JE, Chelly J, Vong LVP, Sy O, Rolin N, Ellrodt O, Monchi M, Vinsonneau C. Does metformin exposure before ICU stay have any impact on patients' outcome? A retrospective cohort study of diabetic patients. Ann Intensive Care. 2017;7(1):116.

21. Green JP, Berger T, Garg N, Suarez A, Hagar Y, Radeos MS, Panacek EA. Impact of metformin use on the prognostic value of lactate in sepsis. Am J Emerg Med. 2012;30(9):1667-73.

22. Park J, Hwang SY, Jo IJ, Jeon K, Suh GY, Lee TR, Yoon H, Cha WC, Sim MS, Carriere KC, et al. Impact of metformin use on lactate kinetics in patients with severe sepsis and septic shock. Shock. 2017:47(5):582-7.

23. van Vught LA, Scicluna BP, Hoogendijk AJ, Wiewel MA, Klein Klouwenberg PM, Cremer OL, Horn J, Nurnberg P, Bonten MM, Schultz MJ, et al. Association of diabetes and diabetes treatment with the host response in critically ill sepsis patients. Crit Care. 2016;20(1):252.

24. Stroup DF, Berlin JA, Morton SC, Olkin I, Williamson GD, Rennie D, Moher D, Becker BJ, Sipe TA, Thacker SB. Meta-analysis of observational studies in epidemiology: a proposal for reporting. Meta-analysis of observational studies in epidemiology (MOOSE) group. Jama. 2000;283(15):2008-12.

25. Baber SR, Deng W, Master RG, Bunnell BA, Taylor BK, Murthy SN, Hyman AL, Kadowitz PJ. Intratracheal mesenchymal stem cell administration attenuates monocrotaline-induced pulmonary hypertension and endothelial dysfunction. Am J Phys Heart Circ Phys. 2007;292(2):H1120-8.

26. Begg $\mathrm{CB}$, Mazumdar M. Operating characteristics of a rank correlation test for publication bias. Biometrics. 1994;50(4):1088-101.

27. Stuck AE, Rubenstein LZ, Wieland D. Bias in meta-analysis detected by a simple, graphical test. Asymmetry detected in funnel plot was probably due to true heterogeneity. BMJ. 1998;316(7129):469 author reply 470-461.
28. Romero SP, Andrey JL, Garcia-Egido A, Escobar MA, Perez V, Corzo R, GarciaDomiguez GJ, Gomez F. Metformin therapy and prognosis of patients with heart failure and new-onset diabetes mellitus. A propensity-matched study in the community. Int J Cardiol. 2013;166(2):404-12.

29. Zhang X, Harmsen WS, Mettler TA, Kim WR, Roberts RO, Therneau TM, Roberts LR, Chaiteerakij R. Continuation of metformin use after a diagnosis of cirrhosis significantly improves survival of patients with diabetes. Hepatology. 2014;60(6):2008-16.

30. Harris K, Smith L. Safety and efficacy of metformin in patients with type 2 diabetes mellitus and chronic hepatitis C. Ann Pharmacother. 2013;47(10):1348-52.

31. Christiansen $\mathrm{C}$, Johansen M, Christensen S, O'Brien JM, Tonnesen $\mathrm{E}_{\text {, }}$ Sorensen $\mathrm{H}$. Preadmission metformin use and mortality among intensive care patients with diabetes: a cohort study. Crit Care. 2013;17(5):R192.

32. Ghavimi H, Sheidaei S, Vaez H, Zolali E, Asgharian P, Hamishehkar H. Metformin-attenuated sepsis-induced oxidative damages: a novel role for metformin. Iran J Basic Med Sci. 2018;21(5):469-75.

33. Kang W, Wang T, Hu Z, Liu F, Sun Y, Ge S. Metformin inhibits Porphyromonas gingivalis lipopolysaccharide-influenced inflammatory response in human gingival fibroblasts via regulating activating transcription factor-3 expression. J Periodontol. 2017;88(10):e169-78,

34. Kim J, Kwak HJ, Cha JY, Jeong YS, Rhee SD, Kim KR, Cheon HG. Metformin suppresses lipopolysaccharide (LPS)-induced inflammatory response in murine macrophages via activating transcription factor-3 (ATF-3) induction. Jiol Chem. 2014;289(33):23246-55.

35. Vaez H, Rameshrad M, Najafi M, Barar J, Barzegari A, Garjani A. Cardioprotective effect of metformin in lipopolysaccharide-induced sepsis via suppression of toll-like receptor 4 (TLR4) in heart. Eur J Pharmacol. 2016;772:115-23.

36. Wu W, Wang S, Liu Q, Shan T, Wang Y. Metformin protects against LPSinduced intestinal barrier dysfunction by activating AMPK pathway. Mol Pharm. 2018;15(8):3272-84.

37. Zhang X, Shang F, Hui L, Zang K, Sun G. The alleviative effects of metformin for lipopolysaccharide-induced acute lung injury rat model and its underlying mechanism. Saudi Pharm J. 2017;25(4):666-70.

38. Wu K, Tian R, Huang J, Yang Y, Dai J, Jiang R, Zhang L. Metformin alleviated endotoxemia-induced acute lung injury via restoring AMPK-dependent suppression of mTOR. Chem Biol Interact. 2018;291:1-6.

39. Gras V, Bouffandeau B, Montravers PH, Lalau JD. Effect of metformin on survival rate in experimental sepsis. Diabetes Metab. 2006;32(2):147-50.

40. Cheng SC, Scicluna BP, Arts RJ, Gresnigt MS, Lachmandas E, GiamarellosBourboulis EJ. Broad defects in the energy metabolism of leukocytes underlie immunoparalysis in sepsis. Nat Immunol. 2016;17(4):406-13.

41. Hui S, Ghergurovich JM, Morscher RJ, Jang C, Teng X, Lu W, Esparza LA, Reya T, Le Z, Yanxiang Guo J, et al. Glucose feeds the TCA cycle via circulating lactate. Nature. 2017;551(7678):115-8.

42. Friesecke $S$, Abel P, Roser M, Felix SB, Runge $S$. Outcome of severe lactic acidosis associated with metformin accumulation. Crit Care. 2010;14(6):R226.

43. Vecchio S, Protti A. Metformin-induced lactic acidosis: no one left behind. Crit Care. 2011;15(1):107.

44. Detaille D, Guigas B, Chauvin C, Batandier C, Fontaine E, Wiernsperger N, Leverve X. Metformin prevents high-glucose-induced endothelial cell death through a mitochondrial permeability transition-dependent process. Diabetes. 2005;54(7):2179-87.

45. Faure P, Wiernsperger N, Polge C, Favier A, Halimi S. Impairment of the antioxidant properties of serum albumin in patients with diabetes: protective effects of metformin. Clin Sci (Lond). 2008;114(3):251-6.

46. Carter AM, Bennett CE, Bostock JA, Grant PJ. Metformin reduces C-reactive protein but not complement factor C3 in overweight patients with type 2 diabetes mellitus. Diabet Med. 2005;22(9):1282-4.

47. Kato Y, Koide N, Komatsu T, Tumurkhuu G, Dagvadorj J, Kato K, Yokochi T. Metformin attenuates production of nitric oxide in response to lipopolysaccharide by inhibiting MyD88-independent pathway. Horm Metab Res. 2010;42(9):632-6.

48. Hardie DG, Ross FA, Hawley SA. AMPK: a nutrient and energy sensor that maintains energy homeostasis. Nat Rev Mol Cell Biol. 2012;13(4):251-62.

49. Madiraju AK, Erion DM, Rahimi Y, Zhang XM, Braddock DT, Albright RA, Prigaro BJ, Wood JL, Bhanot S, MacDonald MJ, et al. Metformin suppresses gluconeogenesis by inhibiting mitochondrial glycerophosphate dehydrogenase. Nature. 2014;510(7506):542-6.

50. Courtois S, Benejat L, Izotte J, Megraud F, Varon C, Lehours P, Bessede E. Metformin can inhibit helicobacter pylori growth. Future Microbiol. 2018;13:1575-83.

51. Malik F, Mehdi SF. Is metformin poised for a second career as an antimicrobial? Diabetes Metab Res Rev. 2018;34(4):e2975. 